\title{
Transient luminous events registered with a ground pinhole
}

\author{
E. Ponce, H. Salazar, O. Martinez. and R. Conde* \\ BUAP, Mexico \\ E-mail: eponcelfcfm.buap.mx
}

\begin{abstract}
The near UV background level at the atmosphere has several sources, such as transient luminous events, trace of micro-meteorites and human activities by example. In order to evaluate the possibility to detect ultra high energy cosmic ray fluorescence signals from the space, it is necessary to measure and monitor this UV background level. Nature of the UV atmospheric transient events is not well known yet and more experimental data are needed. By that, we constructed a fast imaging detector, a pinhole camera. The pinhole camera design fulfills the requirements of FOV, compactness, reliability, and attached to a fast position sensitive detector PSD will allow us to obtain 2d-images, as proposed in the near future space mission TUS (tracking ultraviolet system). In this work we present the shape and energy of some TLEs recorded in the Sierra Negra Volcano near Puebla, Mexico (4300 m.a.s.l).
\end{abstract}

The 34th International Cosmic Ray Conference,

30 July- 6 August, 2015

The Hague, The Netherlands

${ }^{*}$ Speaker. 


\section{Introduction}

One of the important phenomena in night atmosphere, directly related to UHECR measurement, are TLE (transient luminous events) characterized by very bright (energy in UV up to 0.1-1 MJ) short (duration of 1-100 ms) flashes. The first global measurements of UV flashes were done by the Tatiana space detector, but still the important characteristics: the lateral distribution of UV glow in one flash and the energy spectrum of flashes are not measured. In this paper we present a new method of TLE measurement by the imaging pinhole camera. The presented pinhole camera is planned for operation in space experiments devoted to study of processes of electron acceleration in the atmosphere electric discharges and relativistic electron precipitation to the atmosphere from the magnetosphere among others.

\section{Pinhole camera}

The Camera obscura detector is the one of best optical imaging designs due to their simplicity like [1], wide field of view and large deep field such as [2]. We plan to operate it simultaneously with the TUS (Tracking Ultraviolet Setup) fluorescence telescope [3, 4] a space borne mission planed to orbit the earth at $700 \mathrm{Km}$ height. The TUS telescope will register the UHECR track in the upper atmosphere in order to determine the arrival direction and total energy. The camera obscura it will be used to explore the eventual creation of perturbations near to the event that may should trigger some TLEs. This perturbation was considerate as background noise in the tracking of the original particle. The high brightness of TLE may allow us to use the simplest pinhole optics for measuring the image in pixels of UV detector $[5,6]$. The optimal imaging quality in a pinhole camera is achieved if the hole size is equal to the detector pixel size. Our aim is to measure not only the TLE image but also the temporal profile of the image with time resolution of about 64 ms. Today such a fast photo detector is available only as a Multi-Anode Photomultiplier Tube (MaPMT) with some number of pixels. The size of the pixel in MaPMT is of about 2-3 mm. Assuming the camera hole equal to this size and taking the TLE UV intensity and time duration from [3] it is possible to estimate the signals in the pixels of the pinhole camera. Efficiency of the MaPMT pixels to UV is around $20 \%$ for wavelengths $\lambda=300-400 \mathrm{~nm}$ and decreases below $\lambda=300$ $\mathrm{nm}$. For estimate of the TLE image signals the lateral distribution of UV intensity during the event is needed. We assume that UV flash images cover a circular area of $40 \mathrm{~km}$ diameter (as it was measure in a typical TLE by video cameras) with uniform intensity over the circle. So the total UV energy Euv radiated by the TLE correspond to the number of photons of wavelength $\lambda=300$ $400 \mathrm{~nm}$. To improve the detection of TLE, for this project we considered two configurations of the pinhole camera: One short focal distance telescope (SFD); the circle of diameter $40 \mathrm{~km}$ is observed by one pixel and one long focal distance telescope (LFD); the whole $40 \mathrm{~km}$ circle is observed by all pixels of the imaging tracking device for example. In the first option the camera Field of View (FOV) is wide and the signal in one pixel give us a quick estimation of the position of TLE. Full energy released in UV in the atmosphere is determined by the pixel signal. In the second setup a detailed image of the UV flash in space and time is obtained. Both options could be combined in one instrument capable to observe TLE images in the device know as camera obscura, as it was suggested in [1] for EAS Cherenkov light observations, a scheme of the designed camera obscura 


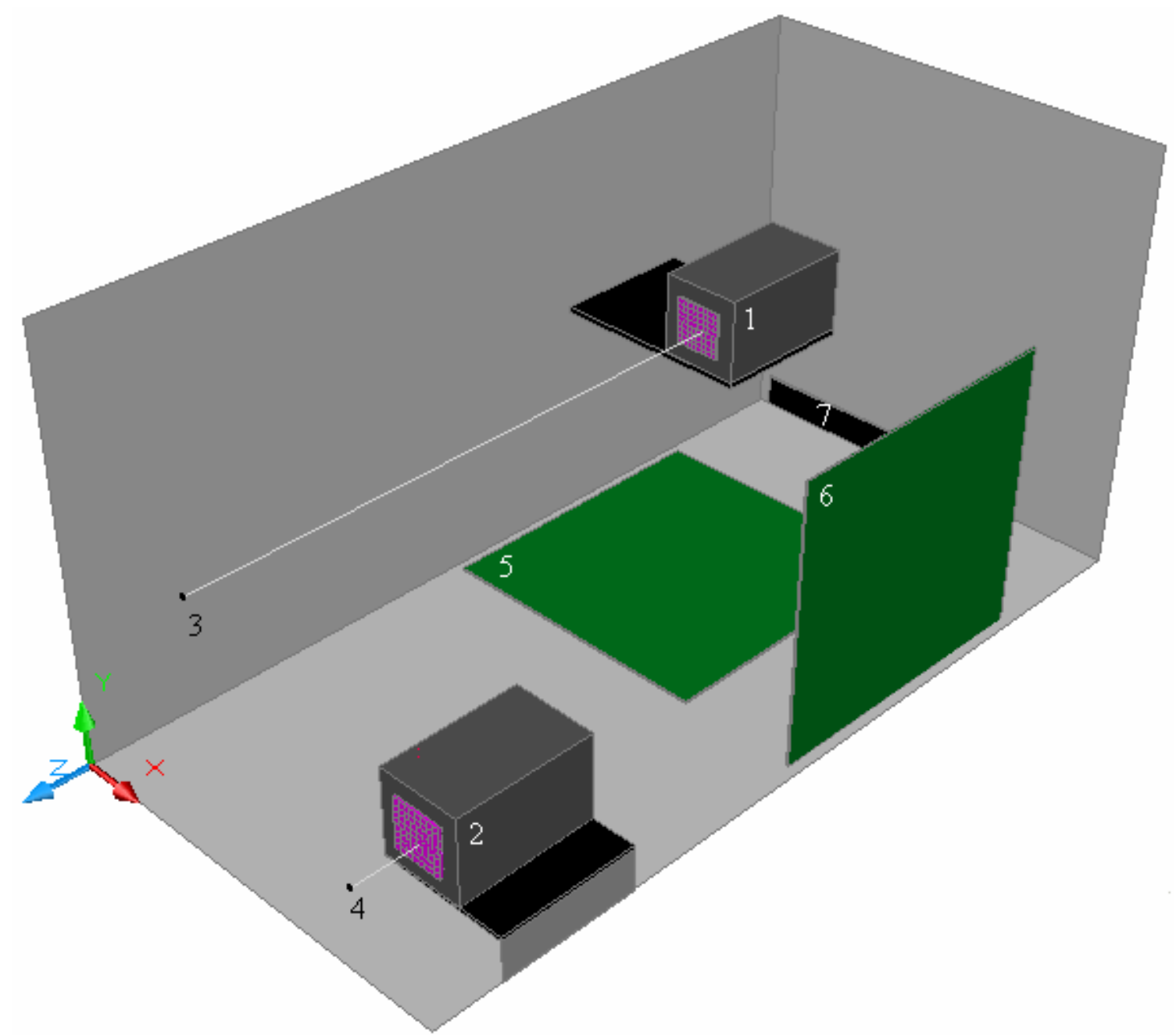

Figure 1: Camera obscura. 1- Long focal distance photo receiver (LFD). 2- Short focal distance photo receiver (SFD). (3 and 4)- Pinholes for each photo receiver. (5 and 6)- Electronic board for each telescope. 7- Communication port.

with two photo detectors it is shown in Figure 1. In our case the design and construction of the camera was made with the following technical parameters: two photo receivers MaPMT model H7546B (Hamamatsu), a matrix of 8 by 8 pixels with a pixel size of $2 \times 2 \mathrm{~mm}$. The holes have the same size. For the Long focal distance configuration (LFD) $\mathrm{f}=20 \mathrm{~cm}$. So with this pixel size in the atmosphere from a distance $\mathrm{R}=500 \mathrm{~km}$., the area observed is $5 \mathrm{kmx} 5 \mathrm{~km}$ for example. For the Short Focal Distance configuration (SFD) $\mathrm{f}=2.5 \mathrm{~cm}$, one pixel of this receiver covers $40 \times 40 \mathrm{~km}$ in the atmosphere. In the test and developing process of the camera obscure detector, as a first stage, we have performed several measurements at the Sierra Negra Volcano (4300 m.a.s.l) due to the small light pollution and less atmospheric absorption.

\section{Camera electronics}

The figure 2 show one of two electronic boards used to the signal processing of each MaPMT 


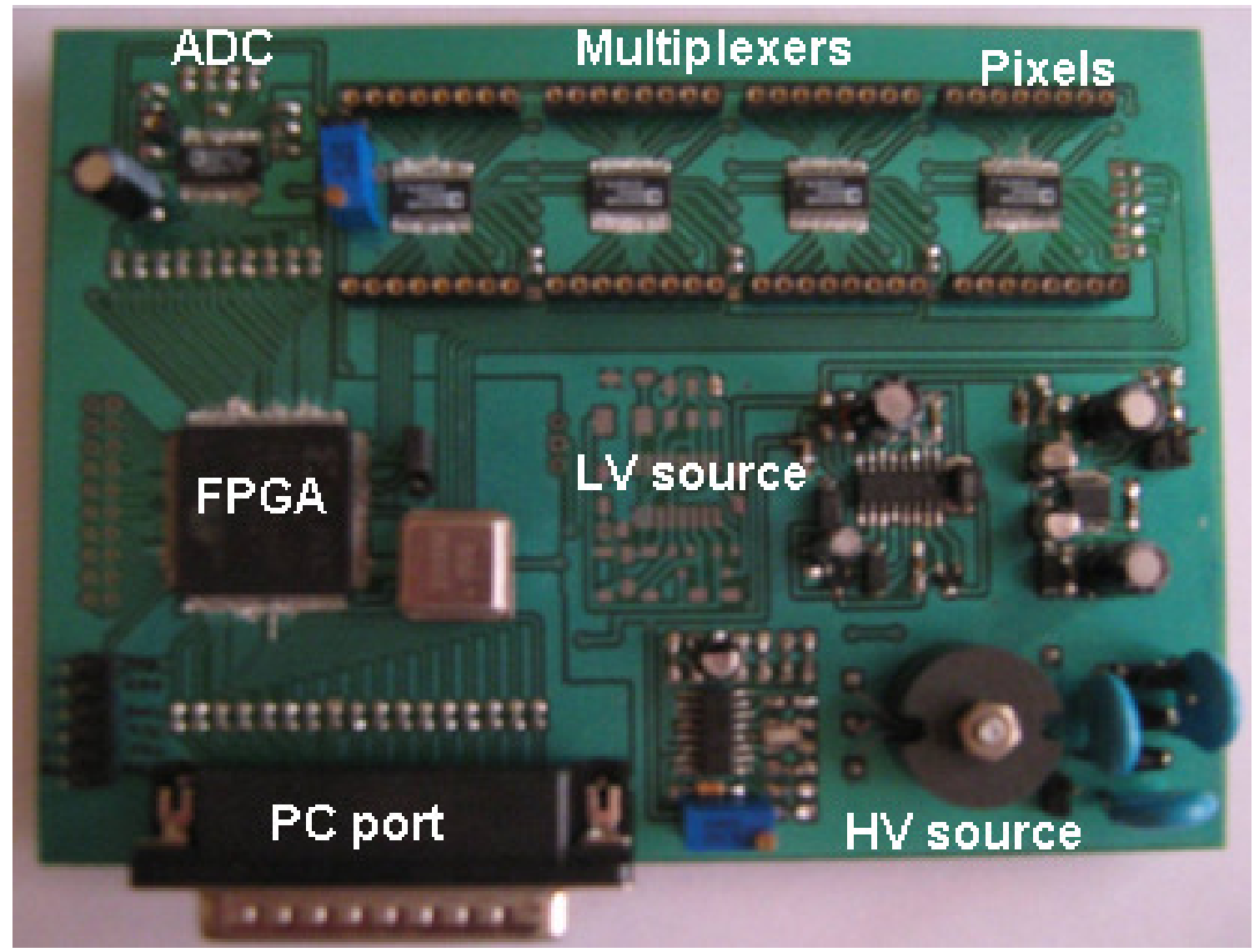

Figure 2: One pinhole camera electronic board.

when a luminous event will appears. Both electronic boards have the same structure in hardware but different configuration according to the parameters of each kind of observed event. Each board have a FPGA Xilinx XCV100 series. This FPGA control the multiplexing and the digitalization of the 64 analog signals from each MaPMT. Also control and monitored the high voltage supplied to the MaPMT in order to protect it if a bright or long lasting event appears. The FPGA stores all the configuration and operation parameters from the PC and communicate it thru parallel port. The FPGA of the SFD is the camera obscure main processor, because controls also the LFD data. The command controls of each FPGA are defined by the user by a graphic programmable interface. With the same software we construct a data base in order to process and plot the registered events. The figure 3, shows the components to multiplex the 64 channels from each MaPMT. Figure 4, it shows the transmission-reception data and commands to the FPGA thru PP port of the PC.

\section{Camera testing}

As a first step in the calibration process of the Camera Obscura, it is necessary to measure the response to a single photo-electron by the MaPMT [7], and use it to convert the signal to physical photon flux. To obtain the single photoelectron response, we have found the optimal operative voltage for PMT. To do this, we checked the single photo-electron (SPE) spectrum as a function of 


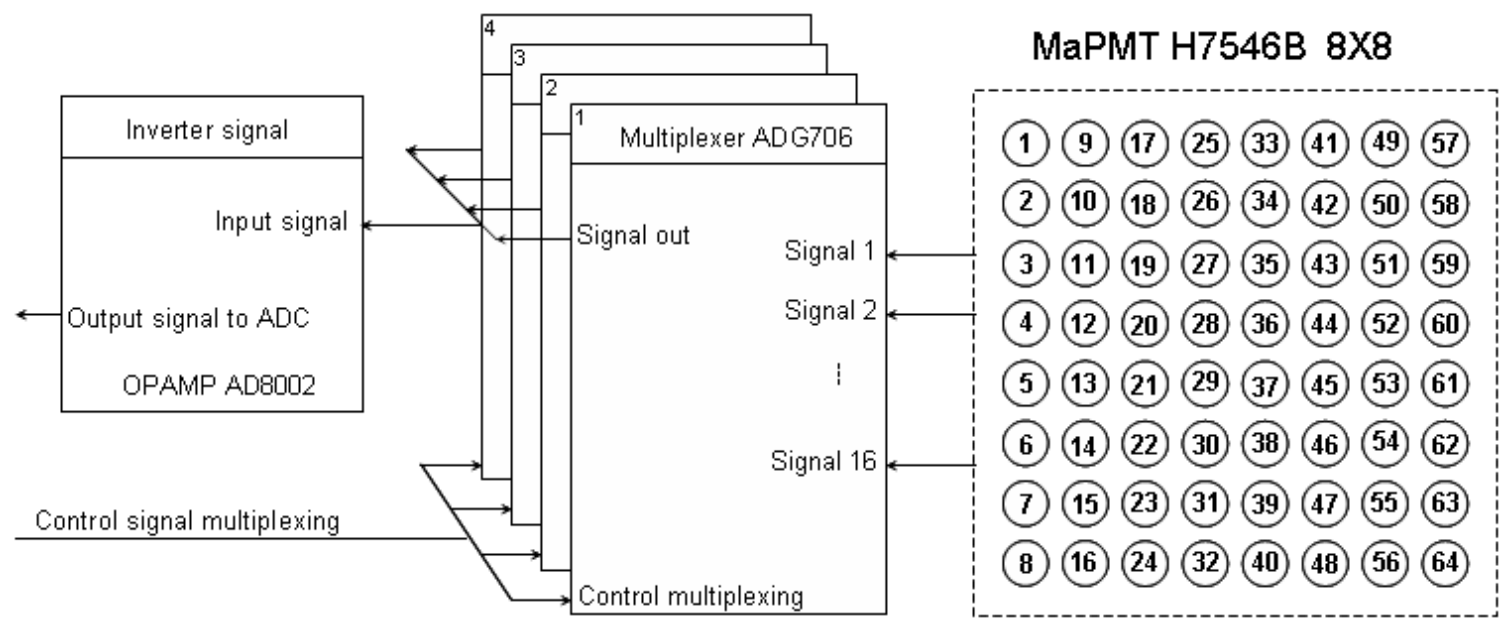

Figure 3: Multiplexing module.

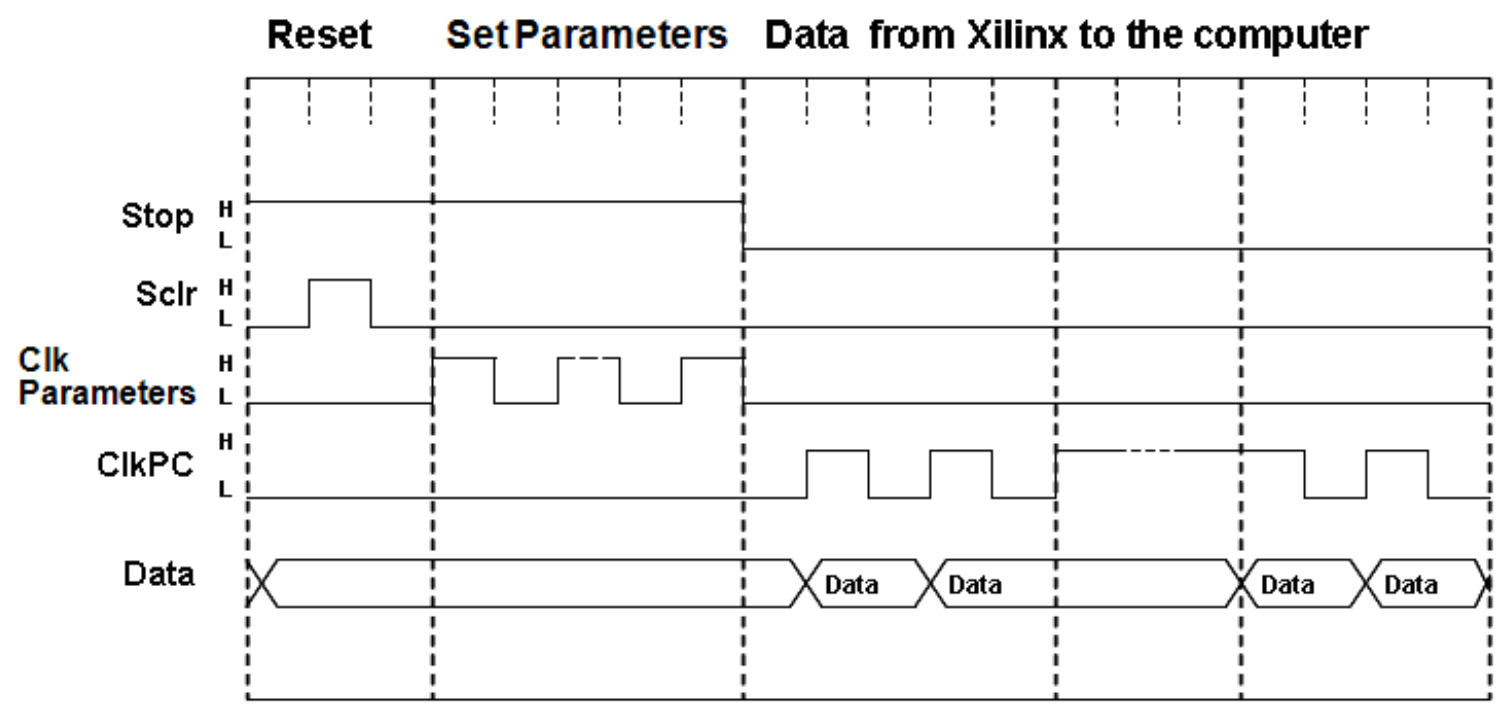

Figure 4: Transmission and reception data and commands thru port PC and FPGA.

supplied voltage with a controlled LED pulse. We selected 950V as operative voltage. From the analysis of 35,000 events, we found the mean charge produced by a single photo-electron response (SPE) and the SPE pulse amplitude distribution. The designed pinhole camera was tested and calibrated measuring the Moon luminosity [8]. By positioning the camera for observing the Moon image in a SFD camera (the Moon angular size $\sim 0.5^{\circ}$ is much less than the pixel FOV $\sim 4.6^{\circ}$ ) it is easy to measure the reference moon luminosity in intensity. The MaPMT registering moon light start working with a constant anode current defined by the ADC code recorded $\mathrm{N}$ of ten bits (the maximal code value is 1024). When code $\mathrm{N}$ is greater than a reference then the current decreases (by less than a factor of 10) in inverse proportion to the square root of the mean value of the anode current. In these operating conditions, one encounters the additional problem of how to control and monitor the gain of the MaPMT to register the most very bright (powerful) phenomena (TLE). 

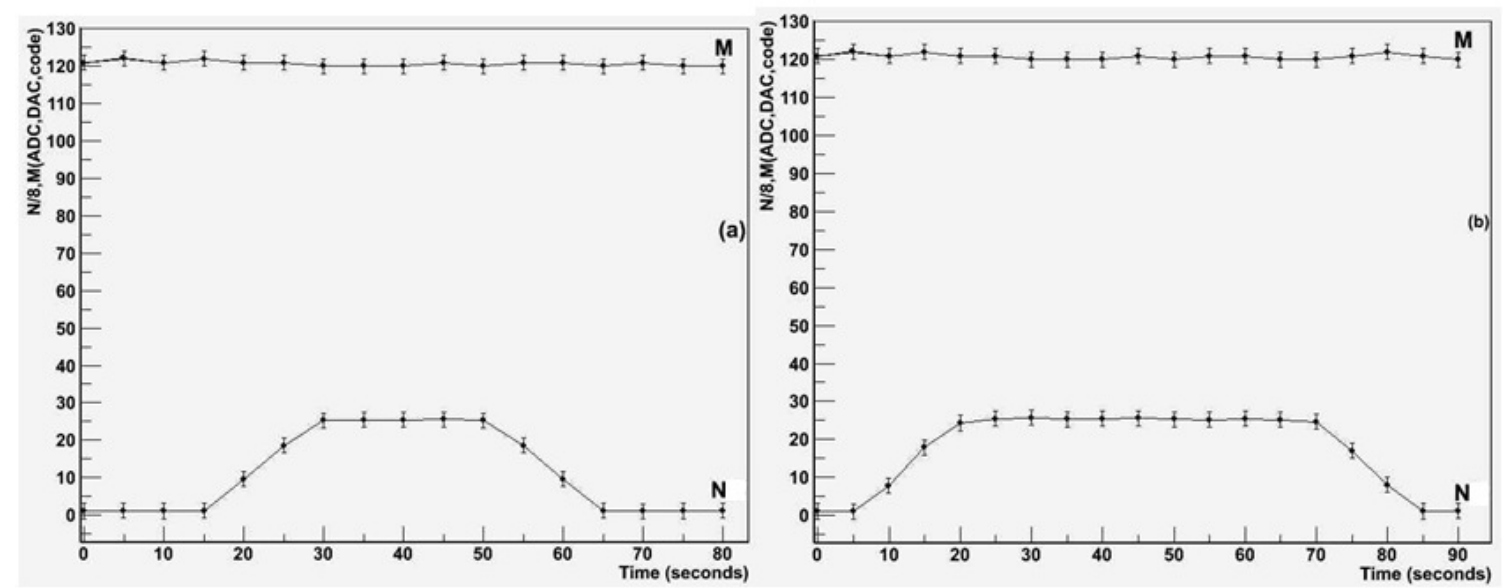

Figure 5: Counts recorded when the Moon image crossing every pixel. a) LFD MaPMT, b) SFD MaPMT. Dots with the error bars are the experimental data, and the continuous curve is the variation of the counts in terms of $\mathrm{N}$ and $\mathrm{M}$ code.

The FPGA controls the MaPMT high voltage power supply by using an eight-digit digital to analog converter (DAC). The supply voltage is proportional to the DAC control code M. The maximal code value is 128 , corresponding to a voltage of $\approx 1000 \mathrm{~V}$. Every minute the automatic control circuit controls the voltage (gain) of MaPMT in correspondence with the illumination of the MaPMT. A useful way to test and calibrate the performance of the pinhole camera, consist in to record the transit of the Moon image crossing every pixel. A telescope mounting base was used to fix the pinhole camera in order to point and track the moon image into a given pixel. In the SFD mode, the crossing time of the MaPMT is about $\sim 60 \mathrm{~s}$. In LFD mode, the crossing time by the MaPMT, will be shorter: $\sim 25 \mathrm{~s}$. In SFD mode, the current will quickly rise to the maximum value and then fall sharply when moon image goes out of the pixel. In LFD mode, the signal from moon will rise and fall continuously having maximum when the moon image is fully inside the limits of the pixel area. Figure 5 shows the code registered in counts $(\mathrm{N}, \mathrm{M})$ in order to plot in the same range (1 to 128) both parameters.

Example of UV TLE event registered (on July 24, 2010 at 2:55 am Mexico time) at mountain Sierra Negra-Mexico (4300 m.a.s.l) is shown in Figures 6 and 7. The event was registered on a thunderstorm developed at a distance of 2 kilometers from camera obscura position, the instrument was observing at 20 degrees from the zenith (because for this angle the absorption is depreciated) and around $4 \pi$. The frequency of multiplexed pixels was of 500 microseconds, the total sampling of 64 pixels were 32 milliseconds. 


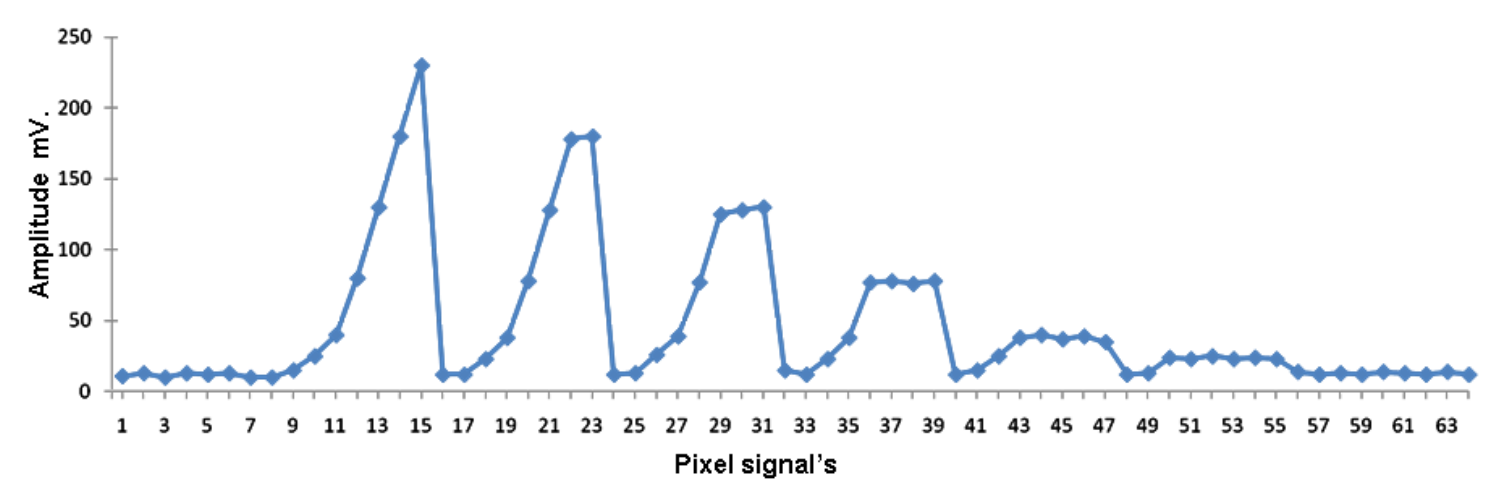

Figure 6: TLE registered with pinhole camera. Signal profile reconstructed with the sequence of 64 pixels multiplexed.

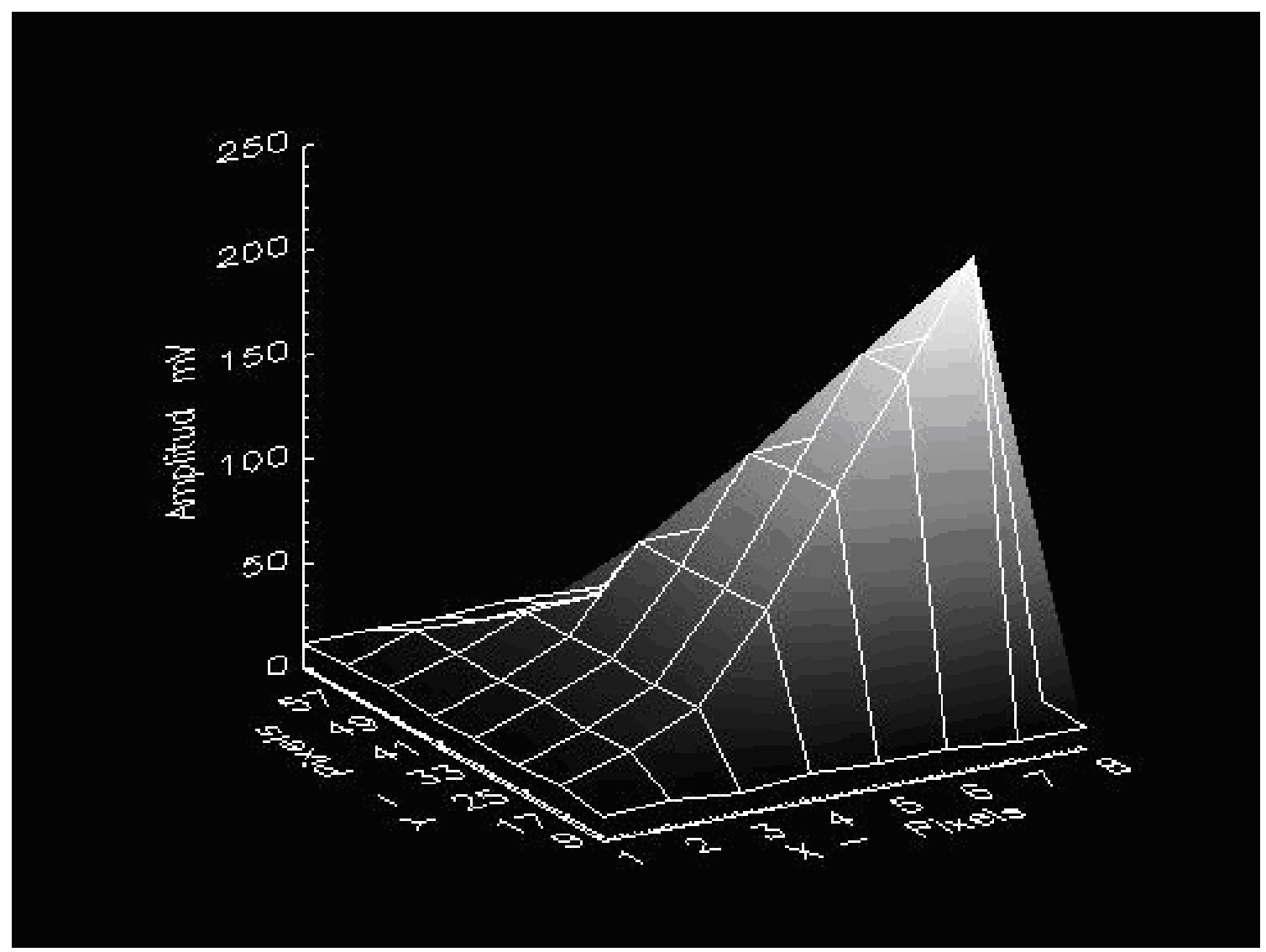

Figure 7: UV TLE registered with pinhole camera. Image reconstructed according the distribution and amplitude registered for each pixels of MaPMT. 


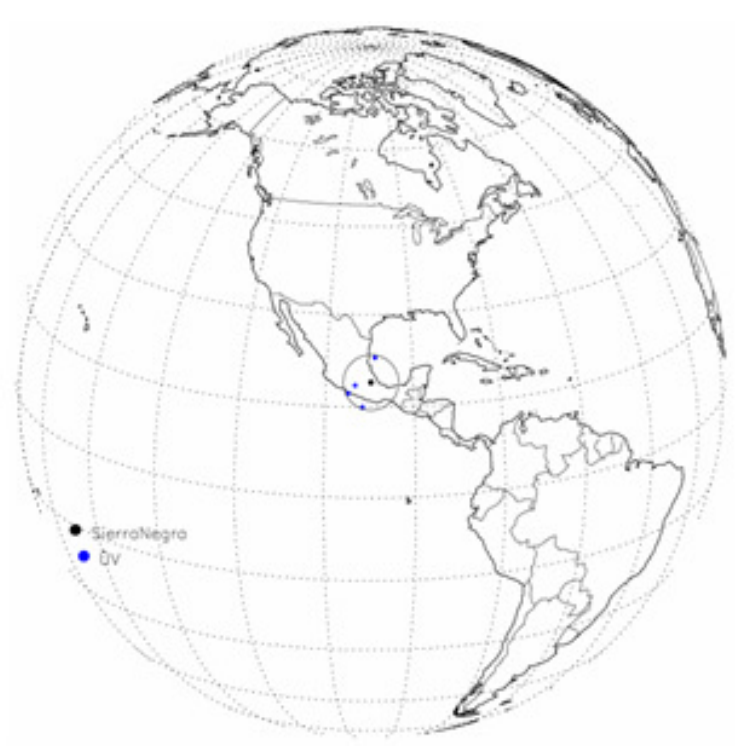

Figure 8: UV TLEs registered onboard Tatyana 2 Satellite, 1000 km. around Sierra Negra-Mexico..

\section{Conclusions}

The pinhole camera design has shown to be a fruitful configuration for studies of background light level distribution presented in the atmosphere. Âă The calibration and performance test at the Mexican mountain Pico de Orizaba âĂŞ Sierra Negra shows that its possible to detect with high confidence level the TLE and UV background light.

\section{References}

[1] G K. Garipov, Camera obscura for observation of EAS images in Cerenkov light, J. Phys. G: Nucl. Part. Phys. 20 Printed in the UK [1994].

[2] G. K. Garipov, Camera obscura network: an array for registration of EAS Cerenkov radiation in the presence of high-intensity sky noise, J. Phys. G: Nucl. Part. Phys. 21 Printed in the UK.

[3] Abrashkin, Space detector TUS for extreme energy cosmic ray study, Nuclear Physics B. 66.

[4] Abrashkin, Updated TUS space fluorescence detector for study of UHECR, Advances in Space Research 41.

[5] Dmitriev, UV radiation from the night-time atmosphere seen from the Universitetsky-TATIANA satellite, in proceedings of AIP $\mathbf{1 1 1 8 .}$

[6] Garipov G.K., Program of transient UV event research at TATIANA-2 satellite, Journal of Geophysical Research A $\mathbf{1 1 5}$.

[7] Y. Kawasaki., Performance of a multi-anode photomultiplier employing a weak electrostatic focusing system (Hamamatsu R8900 series), Nuclear Instruments and Methods in physics research A 564.

[8] E. Ponce., Pinhole camera for study of atmospheric UV flashes as a source of background in the TUS experiment), Nuclear Instruments and Methods in physics research A 639. 